\title{
Expression of TaV Tagged Sindbis Virus (TR339) in Aedes albopictus Cell Lines and Adult Mosquitoes
}

\author{
Jason Saredy and D. Bowers
}

Department of Biology, University of North Florida, Jacksonville FL

Arthropod-borne-viruses (arboviruses) pose a global threat due to their ability to be transmitted by hematophagous insects to vertebrate hosts resulting in a range of serious infectious diseases [1]. Sindbis virus (SINV) is an arbovirus of the genus Alphavirus and the family Togaviridae. This virus is im bibed during bloodfeeding by female mosquitoes in order to enrich their egg production with protein and cholesterol [2]. Bloodmeals are deposited in the mosquito midgut and virions must first cross the gut ('gut barrier') via infection to enter the hemolymph and contact various target tissues [3,4]. The mosquito midgut is a simple column are pithelium surrounded by a basement membrane, peristaltic muscle bundles, nerve fibers, and tracheoles [5].

For a permissive infection route, the virus penetrates the luminal apical aspect, the interface between the bloodmeal and the midgut cells. The bloodmeal typically has two barriers; first is a peritrophic matrix that prevents direct contact of the bloodmeal with the epithelial layer [6] and second the columnar cells that makeup the midgut are covered with microvilli including a complex microvilli-associated network [7].

The purpose of this study was to investigate the use of a tagged virion in the in vitro and in vivo environments. The fluorescent protein GFP was inserted between the Capsid and PE2 in the genome of TR339; SINVTaV-GFP [8]. During virus translation, GFP is attached to PE2, a precursor protein that gets differentially cleaved in the cytoplasm. This virus construct then has the same infectivity and virulence as wild type TR339 [8], and will leave a fluorescent 'path' in infected cells revealing the virus transit.

This study used two Aedes albopictus mosquito cell lines, C7-10 and C6/36, to monitor the infection of stock virus compared with progeny virus. The C7-10 cells were first in fected with SINVTaV-GFP and the supernatant (progeny virus) was collected. Both SINVTaV-GFP and progeny were used to infect C7-10 and C6/36 cells in glass cover-slipped chambers. Images were taken at various time points post-infection (p.i .). Presence of GFP was observed at 6 hours p.i. with confluent presentation at 24 hours p.i. in stock and progeny virus, showing good retention of reporter protein.

We determined that fluorescent quenching and cell leakage of proteins was not observed. Fluorescent quenching was assayed following two challenges; a 24 hour time period with consistent beam exposure and 2 weeks on a countertop exposed to direct sunlight. Secondly cells were fixed with $4 \%$ paraformaldehyde in $0.1 \mathrm{M} \mathrm{Na}$ Cacodylate buffer $(\mathrm{pH} 7.4)$ and imaged at 72 hours for any evidence of cell leakage of fluorescence. 
Ultimately, Ae.aegypti mosquitoes were challenged with a viremic bloodmeal at a titer of $10^{7} \mathrm{PFU} / \mathrm{ml}$ SINVTaV-GFP and midguts were dissected over several days. The presence of GFP was observed at day 3 p.i. as a small foci and day 5 p.i. as a larger foci area. The GFP was observed in the columnar epithelia cells at these time points supporting gut infection [9].

[1] J. Strauss and E. Strauss, Microbiol. Rev. 58 (1994), pp. 491-562.

[2] A. Clements in "The Biology of Mosquitoes", (Chapman and Hall, London) p. 222.

[3] J. Hardy, W. Reeves and R. Sjoren, Am. J. Epidem. 103 (1976), pp. 498-505.

[4] A. Passarelli, Virology 411 (2011), pp. 383-392.

[5] M. Brown, A. Raikhel and A. Lea, Tissue \& Cell 17 Fluorescent (1985), pp. 709-721.

[6] K. Okuda et al, Journal of Insect Physiology 53 (2007), pp. 1307-1315.

[7] H. Zieler et al, The Journal of Experiment Biology 203 (2000), pp. 1599-1611.

[8] C. Sun et al, Journal of Virology 88 (2014), pp. 2035-2046.

[9] The authors acknowledge W. Klimstra of the University of Pittsburgh for providing SINVTaV-GFP in support of this work.

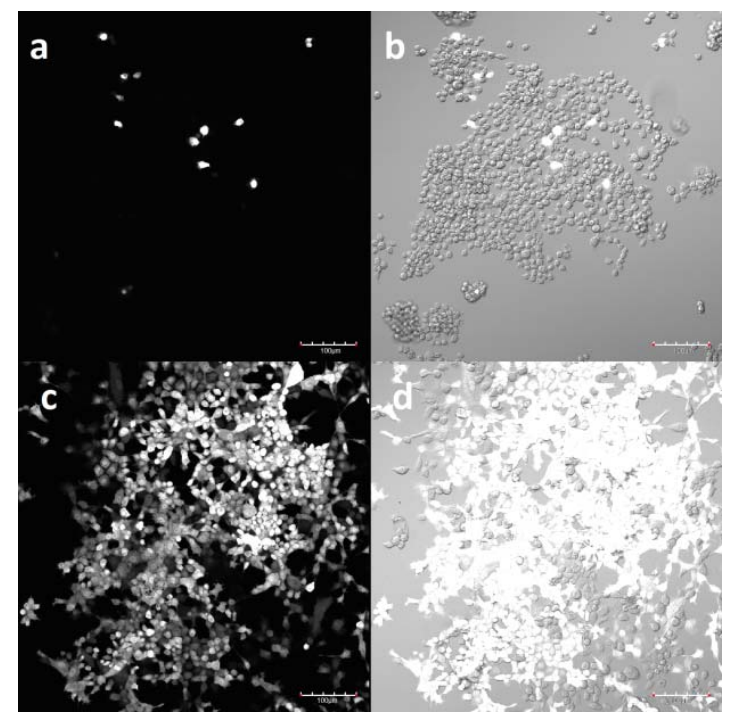

Figure 1. Fluorescent confocal images of C7-10 cells infected with SINVTaV-GFP at $12 \mathrm{hr}$ (a) and $48 \mathrm{hr}$ (c) p.i. and overlay of DIC im ages (b) and (d), respectively. 100 micron scale bar.
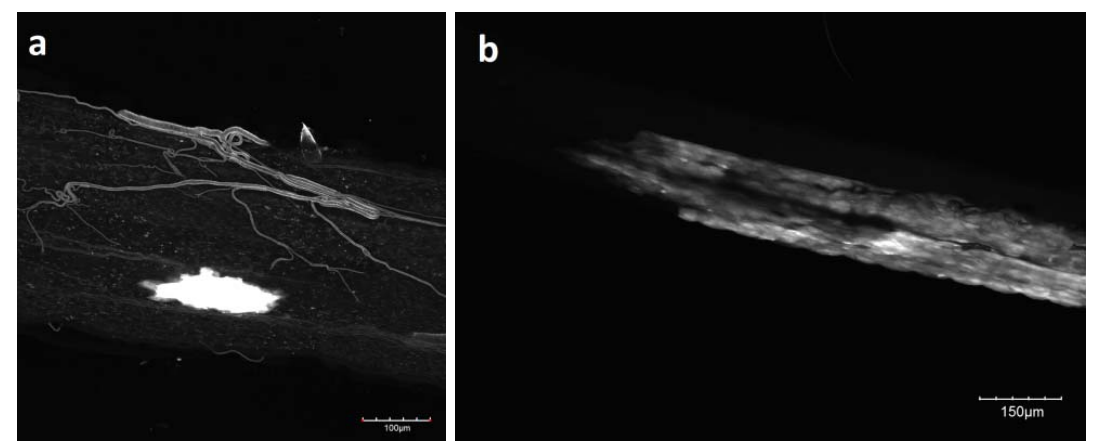

Figure 3. Fluorescent confocal images of Ae. aegypti midguts infected with SINVTaV-GFP. (a) Day 3 p.i. 100 micron scale bar and (b) Day 5 p.i. 150 micron scale bar. 